\title{
Análise do Acesso à Informação Acadêmica entre Estudantes de Medicina Inseridos numa Metodologia Ativa de Aprendizagem
}

\section{Analysis of Access to Academic Information among Medical Students under an Active Learning Methodology}

\author{
Suélem Barros de Lorena ${ }^{l}$ \\ Mateus de Melo Andradel \\ Ângela Melo de Holanda Arcoverde \\ Lycia Siqueira Vilela \\ Luciana Rodrigues Alves da Motal \\ José Eudes de Lorena Sobrinho
}

\section{PALAVRAS-CHAVE}

- Acesso à Informação.

- Comportamento de Busca de Informação.

- Estudantes de Medicina.

- Aprendizagem Baseada em Problemas.
${ }^{I}$ Faculdade Pernambucana de Saúde, Recife, Pernambuco, Brasil

"I Universidade de Pernambuco, Recife, Pernambuco, Brasil 


\section{KEY-WORDS}

- Access to Information.

- Information Search Behavior.

- Medical Students.

- Problem-Based Learning.

\begin{abstract}
Access to academic information has become one of the pillars for the student's role in the learning process, and it is strategic to analyze the behavior and management of available information resources pertinent to the training and excellence of future professionals. The objective of this research was to analyze the behavior reported by medical students of a higher education institution with active learning methodology regarding access to academic information, as well as opinions about the construction of academic knowledge during undergraduate training. A cross-sectional and analytical observational study was conducted with 274 students from the Pernambuco Health College Medical School in Recife, Pernambuco. A specific questionnaire was prepared and validated for the data collection, and subsequently analyzed descriptively using absolute and percentage frequencies for categorical variables and measurements. To evaluate the association between two categorical variables, Pearson's Chi-square test and Fisher's exact test were used. The research project was approved by the ethics committee and respected all ethical requirements. Among those surveyed, 52.8\% used electronic media alone, while $37.7 \%$ indicated that they handled both electronic and print media and 9.4\% cited print media alone. In relation to the forms of study with which the student most identifies, the options confirmed by the majority were "online books (PDF, Word, Epub, etc.)" and "paper books" (81.9\% and 68,3\%, respectively). Regarding questions about the use of electronic databases in their study routine, the majority $(67.9 \%)$ responded positively to the statement; the most commonly cited databases included SciELO (86.7\%), and PubMed (70.6\%). When evaluating access to scientific information among medical students, it was seen that, although most students used electronic databases in their academic routine, more than half had not received training related to bibliographic research techniques; most had learned with practice. Almost all the students surveyed agree on the importance of evidence-based practice in academic routine, which is reported by more than half of the students who, when they do not seek information online, feel less up-to-date.
\end{abstract}

Recebido em: 18/2/19

Aceito em: 7/5/19

\section{INTRODUÇÃO}

O comportamento de busca por informação é o termo utilizado para a procura intencional de conhecimentos a fim de satisfazer algum objetivo, sendo de relevância a identificação dos métodos empregados neste processo. $\mathrm{O}$ indivíduo pode interagir com sistemas de informação manuais (por exemplo, um jornal ou uma biblioteca) ou por computador. O contexto referente às necessidades informacionais é configurado pelo próprio usuário, pelas demandas de seu papel na sociedade e pelas ações desempenhadas no âmbito pessoal e profissional ${ }^{1}$.

Na área acadêmica, os estudantes apresentam diferentes origens, potencialidades, fragilidades, interesses, ambições, sentidos de responsabilidade e níveis de motivação; mostram atitudes desiguais sobre a aprendizagem e o ensino; e têm distintas respostas a ambientes de sala de aula específicos e práticas de instrução ${ }^{2}$. Além disso, a qualidade da informação recebida pelo estudante no curso de graduação também influencia a forma de conduzir os diversos aspectos do cotidiano da sua vida profissional. Uma base sólida de informações adquiridas no decorrer do curso universitário depende da maneira como o estudante é orientado pelos professores ou tutores e da escolha pessoal de inúmeras fontes digitais, materiais e tecnológicas disponíveis atualmente ${ }^{3}$.

Nos últimos anos, as tendências marcantes no campo educacional na área de saúde, especificamente, deram ênfase à aprendizagem centrada no aluno, reconhecendo-o como protagonista do processo da educação, provocando a mudança de uma postura passiva para um papel ativo. Em vista disso, considera-se o papel do professor/tutor como um dos pilares sobre os quais repousa, por exemplo, a Aprendizagem Baseada em Problemas (ABP), metodologia ativa de ensino que desenvolve uma variedade de propósitos educacionais, na qual o educador facilita a aprendizagem por meio de perguntas desafiadoras, direciona a construção do aprendizado e monitora processos educativos individuais e a dinâmica de grupo $^{4}$.

Os alunos das ciências médicas, geralmente, encontram-se em situações que exigem permanente processo de apren- 
dizagem, uso intensivo de tecnologias e enfrentamento de problemas decorrentes de falta de tempo, excessivo volume de informação, escasso e desatualizado acervo da biblioteca das instituições e ausência de treinamento para lidar com ferramentas eletrônicas. Assim, o contexto que envolve os alunos de graduação em Medicina é notadamente singular, e estes representam uma categoria específica, ainda pouco estudada, demandando melhor entendimento ${ }^{5}$.

Em paralelo, a tecnologia de computador permite que a informação seja atualizada com mais frequência, não sendo limitada pelo tamanho e peso de materiais impressos ${ }^{6}$. A diversidade de plataformas de base de dados para pesquisa online, como PubMed, SciELO e EBSCO, constitui um exemplo real da facilidade e possibilidades de acessar o conhecimento pelo meio virtual. Aplicativos, livros em PDF, plataformas online de pesquisa e bibliotecas virtuais se somam às infinidades de formas de comunicação por meio de redes sociais como Facebook, WhatsApp, Skype, entre outras, preenchendo um campo vastíssimo de busca e informação ${ }^{7}$.

Os tradicionais livros e revistas científicas impressos não perderam seu status, embora a rapidez com que se atualizam os torne ineficazes para alguns campos de conhecimento, sendo imperioso que estes se adaptem ao formato virtual de acesso, como evidenciado em diversas editoras ou revistas eletrônicas. Em cursos de graduação em ciências da saúde, a importância das fontes utilizadas para o estudo é imprescindível na hora de equiparar o conhecimento atualizado ao confiável. Os livros físicos, artigos científicos de revistas tradicionais e diretrizes ou consensos de entidades ou órgãos oficiais ainda parecem dominar esta procura pelo conhecimento, mas são inegáveis a força e a rapidez do avanço dos formatos eletrônicos de aplicativos, redes sociais e plataformas de busca de informações médicas baseadas em evidências, que estão conduzindo o aluno a caminhos alternativos ${ }^{8,9}$.

Torna-se necessário conhecer as formas preferidas por alunos do curso de Medicina para a aquisição de conhecimento acadêmico para subsidiar o desenvolvimento de políticas institucionais capazes de proporcionar o fortalecimento do processo de ensino-aprendizagem. O objetivo desta pesquisa foi analisar o comportamento relatado por estudantes de Medicina de uma instituição de ensino superior que adota a metodologia ABP referente ao acesso a informações acadêmicas, bem como opiniões sobre a construção do conhecimento científico durante a graduação.

\section{MÉTODOS}

Trata-se de um estudo observacional, transversal e analítico, desenvolvido entre março de 2017 e julho de 2018 no curso de
Medicina da Faculdade Pernambucana de Saúde, em Recife (PE), Brasil.

O tamanho amostral foi determinado em 274 indivíduos, estratificados em períodos letivos: 44 estudantes do primeiro período, 17 do segundo, 39 do terceiro, 45 do quinto, 43 do sétimo, 45 do nono e 41 do décimo primeiro período. Utilizou-se a amostragem aleatória simples na seleção dos participantes. Para maior sistematização dos resultados referentes aos cruzamentos entre as variáveis, a análise foi feita de acordo com os grupos de períodos em que o aluno se encontrava matriculado, divididos em iniciantes ( $1^{\circ}$ ao $4^{\circ}$ período), intermediários ( $5^{\circ}$ ao $8^{\circ}$ período) e avançados $\left(9^{\circ}\right.$ ao $12^{\circ}$ período).

A coleta de dados foi realizada em duas fases: a primeira correspondeu à validação semântica do questionário estruturado com dez estudantes que não participaram do estudo, a fim de avaliar a clareza e a pertinência das perguntas e a adequação da escala de resposta para possíveis correções, antes de sua aplicação no estudo. Após a validação semântica do questionário, foi realizada a segunda fase da coleta, em que os participantes responderam ao questionário autoaplicável.

Cada questionário tinha quatro blocos: o primeiro continha a identificação do perfil sociodemográfico da amostra selecionada; o segundo reconhecia os dados dos estudantes acerca de sua rotina diária de estudos acadêmicos e da busca por informação; o terceiro seguiu a Escala de Likert com cinco opções de respostas para as sentenças - discordo totalmente, discordo, sem opinião, concordo e concordo totalmente-, contendo assertivas acerca da opinião sobre o acesso à informação e como o estudante avalia o impacto dessa busca por informações online na vida acadêmica; o quarto bloco era indicado apenas para os estudantes que realizam pesquisas em bases de dados eletrônicas para identificar a frequência e o nível de conhecimento deles a respeito desse meio de pesquisa.

Os dados foram analisados descritivamente por meio de frequências absolutas e percentuais para as variáveis categóricas e das medidas: média, desvio padrão e mediana da variável idade. Para avaliar associação entre duas variáveis categóricas foi usado o Teste Qui-Quadrado de Pearson ou o Teste Exato de Fisher quando a condição para utilização do Teste Qui-Quadrado não foi verificada. A margem de erro empregada na decisão dos testes estatísticos foi de 5\%. Os dados foram digitados na planilha MS Excel 2016, e o programa utilizado para obtenção dos cálculos estatísticos foi o IMB SPSS na versão 23 .

Este estudo está rigorosamente baseado nas Resoluções 466/2012 e 510/2016 do Conselho Nacional de Saúde (CNS), e o projeto de pesquisa foi analisado e aprovado pelo Comitê de Ética em Pesquisa com Seres Humanos da Fa- 
culdade Pernambucana de Saúde, sob o número de CAAE: 69974017.1.0000.5569. Todos os participantes foram informados sobre os objetivos da pesquisa e foram orientados quanto ao sigilo das respostas do questionário. Após concordarem com o Termo de Consentimento Livre e Esclarecido (TCLE), os participantes responderam ao questionário autoaplicável. Não há conflito de interesses econômicos ou por vínculos empregatícios.

\section{RESULTADOS}

O total de alunos participantes da pesquisa foi de $n=265$. Destes, a maioria era do sexo feminino $(70,6 \%)$, solteiros $(96,2 \%)$ e não possuíam filhos (96,2\%). A faixa etária variou de 18 a 40 anos, e aproximadamente metade do grupo (50,2\%) tinha entre 20 e 23 anos. A maioria não possuía vínculo empregatício $(93,6 \%)$ ou outra graduação $(91,7 \%)$. O grupo de alunos iniciantes ( $1^{\circ}$ ao $4^{\circ}$ período do curso) prevaleceu $(37,0 \%)$, seguido pelo grupo intermediário ( $5^{\circ}$ ao $8^{\circ}$ período) com $33,2 \%$ e avançado ( $9^{\circ}$ ao $12^{\circ}$ período) com $29,8 \%$.

Em relação ao meio de acesso à informação mais utilizado, a resposta mais frequente correspondeu aos meios eletrônicos (52,8\%). Apenas 9,4\% citaram os meios impressos, e os $37,7 \%$ restantes utilizavam conjuntamente os meios impressos e eletrônicos. Diante dos questionamentos acerca dos meios de estudo com que mais se identificam, mais da metade dos alunos assinalou "livros online (PDF, Word, Epub, etc.)" e "livros em papel" (81,9\% e 68,3\%, respectivamente). Apenas $3,8 \%$ dedicam menos de cinco horas semanais a esse meio de estudo, sendo de cinco a dez horas o maior percentual assinalado (36,2\%). A maioria $(83,8 \%)$ considera que as informações recebidas pelo meio escolhido como prioritário são atualizadas e $92,5 \%$ afirmaram que se sentiam confiantes em utilizar de forma eficaz o conhecimento adquirido pelo determinado meio.

Aproximadamente metade dos estudantes (54,3\%) informou que a instituição preconiza algum tipo de meio de estudo e apenas $43,8 \%$ se preocupam com direitos autorais e referências bibliográficas. Oitenta e seis alunos já frequentaram algum curso de atualização em recursos digitais (32,5\%) e 252 pretendem realizar ou já realizaram algum projeto de pesquisa científica (95,1\%). Na questão "Qual(is) dos interesses intrínsecos abaixo o motiva a procurar por informações acadêmicas?", os percentuais mais significativos das respostas foram "dúvidas ou insegurança" (77,7\%) e "curiosidade" (74,0\%). As respostas mais frequentes à questão "Durante uma consulta à literatura, o que poderia impedi-lo de concluí-la?" foram: dificuldade em localizar documentos pertinentes $(57,7 \%)$, inexistência de uma biblioteca de fácil acesso ou com acervo e serviços adequados
$(49,4 \%)$, falta de tempo $(41,9 \%)$, baixa velocidade da internet (20,8\%), não dispor de computador $(20,4 \%)$ e barreiras linguísticas $(19,2 \%)$.

Entre os 180 pesquisados que afirmaram utilizar bases de dados eletrônicas em sua rotina acadêmica de estudos $(67,9 \%)$, as mais citadas foram: SciELO (86,7\%), PubMed (70,6\%) e Medline $(30,0 \%)$, e as outras quatro respostas possíveis (EBSCO, Bireme, Lilacs e Outra) tiveram percentuais que variaram de $11,1 \%$ a $23,3 \%$.

As opiniões dos grupos selecionados (iniciante, intermediário e avançado) acerca dos meios de estudo com que os estudantes mais se identificam mostraram que os que responderam sim para resumos acadêmicos extraoficiais de colegas acadêmicos $(17,4 \%)$ se reduziram com o avançar da graduação, sendo $27,6 \%$ no grupo de iniciantes ( $1^{\circ}$ ao $\left.3^{\circ}\right), 18,2 \%$ no grupo de intermediários ( $5^{\circ}$ ao $7^{\circ}$ ) e $3,8 \%$ entre os alunos do grupo dos avançados ( $9^{\circ}$ ao $\left.11^{\circ}\right)$. Os que afirmaram pesquisar em sites em geral na internet (17\%) foram 33,7\% no grupo iniciante e variaram de $5,1 \%$ a $9,1 \%$ nos outros dois grupos; dos $11,7 \%$ que citaram plataforma de saúde baseada em evidências (Dynamed/UpToDate) foram 32,9\% no grupo avançado e variaram de $1,0 \%$ a $4,5 \%$ nos outros dois grupos.

Os estudantes que percebem que estarão menos atualizados se não estudarem conteúdos online foi mais elevado no grupo avançado $(67,1 \%)$ e menos elevado no grupo intermediário (40,9\%). Já o percentual de alunos que costuma pegar livros emprestados na biblioteca para estudar foi menos elevado no grupo avançado (46,8\%) e mais elevado no grupo iniciante $(91,8 \%)$.

Dados referentes à relação entre os estudantes pesquisados e os meios de acesso à informação encontram-se descritos na Tabela 1. A Tabela 2 expõe a comparação entre os grupos de estudantes iniciantes, intermediários e avançados a respeito dos meios de estudo prioritários. Informações sobre a utilização de bases de dados na rotina acadêmica de estudos podem ser obtidas na Tabela 3. Por fim, a opinião dos estudantes pode ser analisada, por grupos na Tabela 4.

\section{DISCUSSÃO}

Visando comparar as opiniões dos acadêmicos quanto às preferências de meios de estudo rotineiros e ao treinamento em recursos digitais, foram confrontados os resultados desta pesquisa com outra realizada entre estudantes de Medicina da Faculdade de Ciências de Saúde da Universidade de Zimbabwe ${ }^{10}$, a qual demonstrou que a maior parte $(85 \%)$ dos alunos pesquisados tinha acesso a recursos médicos digitais e impressos, e, destes, mais da metade (54\%) preferiu livros impressos. Tal pesquisa corrobora o presente estudo, no qual a 


\begin{tabular}{|c|c|c|}
\hline $\begin{array}{c}\text { TABELA } 1 \\
\text { Relação dos estudantes pesquisados cc } \\
\text { os meios de acesso à informação }\end{array}$ & & \\
\hline Variável & n & $\%$ \\
\hline Meios de estudos prioritários* & & \\
\hline Livros online (PDF, Word, EPUB, etc.) & 217 & 81,9 \\
\hline Livros em papel & 181 & 68,3 \\
\hline $\begin{array}{l}\text { Artigos científicos on-line (PubMed, EBSCO, SciELO } \\
\text { etc.) }\end{array}$ & 98 & 37,0 \\
\hline Apostilas de cursinhos para residência & 76 & 28,7 \\
\hline Diretrizes ou condutas de órgãos oficiais de saúde & 70 & 26,4 \\
\hline $\begin{array}{l}\text { Resumos acadêmicos extra-oficiais (colegas } \\
\text { acadêmicos) }\end{array}$ & 46 & 17,4 \\
\hline Sites em geral da Internet & 45 & 17,0 \\
\hline $\begin{array}{l}\text { Plataforma de saúde baseada em evidências } \\
\text { (Dynamed/UpToDate) }\end{array}$ & 31 & 11,7 \\
\hline $\begin{array}{l}\text { Artigos científicos em papel (Revistas, Jornais } \\
\text { Científicos etc.) }\end{array}$ & 18 & 6,8 \\
\hline Aplicativos de saúde & 5 & 1,9 \\
\hline $\begin{array}{l}\text { Qual(is) dos interesses intrínsecos abaixo o motiva a } \\
\text { procurar por informações acadêmicas?* }\end{array}$ & & \\
\hline Dúvidas ou insegurança & 206 & 77,7 \\
\hline Curiosidade & 196 & 74,0 \\
\hline Receio de cometer erro & 148 & 55,8 \\
\hline Interesse em pesquisas e publicações & 110 & 41,5 \\
\hline Outros & 12 & 4,5 \\
\hline $\begin{array}{l}\text { Durante uma consulta a literatura, o que poderia } \\
\text { impedi-lo de concluí-la?** }\end{array}$ & & \\
\hline $\begin{array}{l}\text { Inexistência de uma biblioteca de fácil acesso ou com } \\
\text { acervo e serviços adequados }\end{array}$ & 131 & 49,4 \\
\hline Dificuldade em localizar documentos pertinentes & 153 & 57,7 \\
\hline Não dispor de computador & 54 & 20,4 \\
\hline Não dispor de coleção particular & 26 & 9,8 \\
\hline Barreiras lingüísticas & 51 & 19,2 \\
\hline Baixa velocidade da Internet & 55 & 20,8 \\
\hline $\begin{array}{l}\text { Não saber manejar os diferentes recursos informacionais } \\
\text { eletrônicos (Internet, bases de dados, etc.) }\end{array}$ & 42 & 15,8 \\
\hline Falta de tempo & 111 & 41,9 \\
\hline Outros obstáculos & 11 & 4,2 \\
\hline Nenhum obstáculo & 11 & 4,2 \\
\hline
\end{tabular}

maior parte da amostra $(57,7 \%)$ também possuía acesso à literatura científica, incluída neste conjunto de recursos médicos.

Ainda em consonância com a pesquisa citada, nos resultados encontrados neste trabalho, com relevância estatisticamente significativa ( $\mathrm{p}<0,001$ ), está presente o dado de que mais de $50 \%$ dos estudantes (68,3\%) se identifica com os livros em papel. Esta constatou ainda que a maioria dos participantes $(70 \%)$ afirmou não ter recebido treinamento em medicina baseada em evidências. Os resultados corroboram ainda o questionamento acerca de treinamento em técnicas de pesqui- sa bibliográfica (medicina baseada em evidências), em que a maioria da amostra do estudo negou tê-lo recebido.

Nos questionários aplicados na Faculdade de Ciências de Saúde da Universidade de Zimbabwe ${ }^{10}$, as bases de dados Pubmed e Medline, analisadas conjuntamente, possuíam alta popularidade (69\%) entre os estudantes. No presente estudo, os questionários aplicados concordam em parte, visto que apenas a base de dados Pubmed obteve alta popularidade, sendo utilizada na rotina acadêmica de $70,6 \%$ dos alunos, enquanto a Medline corresponde a apenas 30\% destes.

Em relação às atitudes dos estudantes na utilização de bases de dados, estes achados foram comparados com os de um estudo efetuado com estudantes de Medicina da Escola Multicampi de Ciências Médicas do Rio Grande do Norte (UFRN) ${ }^{11}$, que apresenta a ABP como método de aprendizagem. Foi constatado que na UFRN, apesar de os alunos usarem mais a internet do que o acervo da biblioteca para realizar as atividades acadêmicas, quando precisam de informações científicas confiáveis, apenas 33\% relataram pesquisa em bases de dados, ao passo que o grupo majoritário de alunos (68\%) da Faculdade Pernambucana de Saúde utiliza bases de dados eletrônicas em sua rotina acadêmica. Assim, à medida que se realiza pesquisa científica, o que se busca é o conhecimento que possa subsidiar novas tecnologias e aplicações práticas, a fim de se gerar um efeito tangível em determinado campo de ação humana. Desta forma, evidencia-se a importância dessa procura por informações científicas no contexto da formação médica, bem como seu incremento no exercício na área da saúde ${ }^{12}$.

Esse mesmo estudo com alunos do curso de graduação em Medicina da UFRN ${ }^{11}$ expôs que $70 \%$ deles sentiam dificuldades em realizar pesquisas em bases de dados especializadas. Entre as principais dificuldades estavam o uso de técnicas de pesquisa, que incluem operadores booleanos, busca avançada e filtros (53\%); na atual amostra, das pessoas que utilizam bases de dados na rotina acadêmica de estudos, 30\% têm dificuldade no manejo dos recursos nas fontes eletrônicas. Ainda na pesquisa anterior, $19 \%$ dos alunos apresentavam dificuldade na definição dos termos de busca, que incluem o uso de descritores e palavras-chave. Em nosso estudo, um número maior de estudantes (43,3\%) assinalou que encontrar palavras-chave para uma boa estratégia de busca seria um problema em relação à busca por literatura científica nas fontes eletrônicas. Tais resultados demonstram que ainda há dificuldades significativas dos alunos em manusear bases de dados em pesquisa nos meios eletrônicos.

Nesse contexto, diante da predominância do idioma inglês na produção científica da área de saúde, nosso estudo verificou que apenas 19,2\% dos alunos concordaram em que a barreira 


\section{TABELA 2}

Comparativo entre os grupos de estudantes iniciantes, intermediários e avançados a respeito dos meios de estudo prioritários

\begin{tabular}{|c|c|c|c|c|c|c|c|c|c|}
\hline \multirow{3}{*}{ Meios de estudo prioritários } & \multicolumn{6}{|c|}{ Grupo de períodos } & & & \multirow{3}{*}{ Valor de $\mathrm{p}$} \\
\hline & \multicolumn{2}{|c|}{$1^{\circ}$ ao $3^{\circ}$} & \multicolumn{2}{|c|}{$5^{\circ}$ ao $7^{\circ}$} & \multicolumn{2}{|c|}{$9^{\circ}$ ao $11^{\circ}$} & \multicolumn{2}{|c|}{ Grupo total } & \\
\hline & $\mathrm{n}$ & $\%$ & $\mathrm{n}$ & $\%$ & $\mathrm{n}$ & $\%$ & $\mathrm{n}$ & $\%$ & \\
\hline TOTAL & 98 & 100,0 & 88 & 100,0 & 79 & 100,0 & 265 & 100,0 & \\
\hline Livros online (PDF, Word, EPUB, etc.) & & & & & & & & & $\mathrm{p}^{(1)}<0,001^{*}$ \\
\hline Sim & 83 & 84,7 & 82 & 93,2 & 52 & 65,8 & 217 & 81,9 & \\
\hline Não & 15 & 15,3 & 6 & 6,8 & 27 & 34,2 & 48 & 18,1 & \\
\hline Livros em papel & & & & & & & & & $\mathrm{p}^{(1)}<0,001^{*}$ \\
\hline Sim & 81 & 82,7 & 63 & 71,6 & 37 & 46,8 & 181 & 68,3 & \\
\hline Não & 17 & 17,3 & 25 & 28,4 & 42 & 53,2 & 84 & 31,7 & \\
\hline Artigos científicos on-line (PubMed, EBSCO, SciELO etc.) & & & & & & & & & $\mathrm{p}^{(1)}=0,631$ \\
\hline Sim & 38 & 38,8 & 29 & 33,0 & 31 & 39,2 & 98 & 37,0 & \\
\hline Não & 60 & 61,2 & 59 & 67,0 & 48 & 60,8 & 167 & 63,0 & \\
\hline Apostilas de cursinhos para residência & & & & & & & & & $\mathrm{p}^{(1)}<0,001^{*}$ \\
\hline Sim & 8 & 8,2 & 23 & 26,1 & 45 & 57,0 & 76 & 28,7 & \\
\hline Não & 90 & 91,8 & 65 & 73,9 & 34 & 43,0 & 189 & 71,3 & \\
\hline Diretrizes ou condutas de órgãos oficiais de saúde & & & & & & & & & $\mathrm{p}^{(1)}<0,001^{*}$ \\
\hline Sim & 12 & 12,2 & 31 & 35,2 & 27 & 34,2 & 70 & 26,4 & \\
\hline Não & 86 & 87,8 & 57 & 64,8 & 52 & 65,8 & 195 & 73,6 & \\
\hline Resumos acadêmicos extraoficiais (colegas acadêmicos) & & & & & & & & & $\mathrm{p}^{(1)}<0,001^{*}$ \\
\hline Sim & 27 & 27,6 & 16 & 18,2 & 3 & 3,8 & 46 & 17,4 & \\
\hline Não & 71 & 72,4 & 72 & 81,8 & 76 & 96,2 & 219 & 82,6 & \\
\hline Sites em geral da Internet & & & & & & & & & $\mathrm{p}^{(1)}<0,001^{*}$ \\
\hline Sim & 33 & 33,7 & 8 & 9,1 & 4 & 5,1 & 45 & 17,0 & \\
\hline Não & 65 & 66,3 & 80 & 90,9 & 75 & 94,9 & 220 & 83,0 & \\
\hline Plataforma de saúde baseada em evidências (Dynamed/UpToDate) & & & & & & & & & $\mathrm{p}^{(1)}<0,001^{*}$ \\
\hline Sim & 1 & 1,0 & 4 & 4,5 & 26 & 32,9 & 31 & 11,7 & \\
\hline Não & 97 & 99,0 & 84 & 95,5 & 53 & 67,1 & 234 & 88,3 & \\
\hline Artigos científicos em papel (Revistas, Jornais Científicos etc.) & & & & & & & & & $\mathrm{p}^{(1)}=0,783$ \\
\hline Sim & 8 & 8,2 & 5 & 5,7 & 5 & 6,3 & 18 & 6,8 & \\
\hline Não & 90 & 91,8 & 83 & 94,3 & 74 & 93,7 & 247 & 93,2 & \\
\hline Quanto tempo, por semana, dedica a esse meio de estudo? & & & & & & & & & $\mathrm{p}^{(2)}<0,001^{*}$ \\
\hline Menos de 5 horas & - & - & 3 & 3,4 & 7 & 8,9 & 10 & 3,8 & \\
\hline 5 a 10 horas & 24 & 24,5 & 32 & 36,4 & 40 & 50,6 & 96 & 36,2 & \\
\hline 11 a 15 horas & 27 & 27,6 & 28 & 31,8 & 16 & 20,3 & 71 & 26,8 & \\
\hline Mais de 15 horas & 47 & 48,0 & 25 & 28,4 & 16 & 20,3 & 88 & 33,2 & \\
\hline Considera que as informações recebidas por esse meio são atualizadas? & & & & & & & & & $\mathrm{p}^{(1)}=0,182$ \\
\hline Sim & 81 & 82,7 & 70 & 79,5 & 71 & 89,9 & 222 & 83,8 & \\
\hline Não & 17 & 17,3 & 18 & 20,5 & 8 & 10,1 & 43 & 16,2 & \\
\hline $\begin{array}{l}\text { Se sente confiante em utilizar o conhecimento adquirido por esse } \\
\text { determinado meio de forma eficaz? }\end{array}$ & & & & & & & & & $\mathrm{p}^{(1)}=0,083$ \\
\hline Sim & 92 & 93,9 & 77 & 87,5 & 76 & 96,2 & 245 & 92,5 & \\
\hline Não & 6 & 6,1 & 11 & 12,5 & 3 & 3,8 & 20 & 7,5 & \\
\hline
\end{tabular}

(*) Associação significativa ao nível de 5,0\%

(1) Através do teste Qui-quadrado de Pearson.

(2) Através do teste Exato de Fisher. 


\section{TABELA 3}

\section{Avaliação das questões relacionadas à utilização de base de dados na rotina acadêmica de estudos}

Variável

Utiliza bases eletrônicas de dados na sua rotina acadêmica de estudos?

Sim

n

180

67,9

Não

TOTAL

Ao fazer pesquisas científicas, como você aprendeu as técnicas da pesquisa bibliográfica? ${ }^{(1)}$

Aprendeu com a prática

Recebeu orientação ou treinamento de um(a) professor(a) de recursos digitais durante a graduação

Aprendeu em tutoriais ou na "Ajuda" das próprias bases de dados

Foi tema abordado na graduação como parte de uma disciplina

Recebeu orientação ou treinamento de um(a) bibliotecário(a)

Outro modo

Frases que descrevem diferentes estágios no manejo de bases de dados eletrônicos ${ }^{(1)}$

Utiliza os filtros possíveis durante sua pesquisa, selecionando os trabalhos científicos que tem perfil compatível com o 111 seu interesse de busca

Escreve palavras que você considera importantes para o tema na primeira caixa de diálogo que aparece

Combina diversas palavras que você considera importantes para o tema com operadores booleanos (AND, OR, NOT)

Monta estratégias com descritores (MeSH e DeCS) e qualificadores, realizando apenas pesquisas avançadas ${ }^{(1)}$

Com que frequência semanal você acessa uma base de dados eletrônicas?

Raramente

Ao menos um vez por semana

Pelo menos duas vezes por semana

Pelo menos três vezes na semana

Diariamente

Somente quando necessário a pesquisas científicas

Quais problemas mais encontrados em relação à busca por literatura científica nas fontes eletrônicas? ${ }^{(1)}$

Encontrar palavras-chave adequadas para uma boa estratégia de busca

Falta de tempo para se dedicar à busca

Selecionar entre a grande quantidade de documentos recuperados nas buscas

Custo financeiro dos documentos

Dificuldade no manejo dos recursos

Custo financeiro da conexão com a Internet

Qual(is) fatores prioriza para selecionar documentos que gostaria de ler para sua vida acadêmica? ${ }^{(1)}$

Texto completo ser gratuito

Ano de publicação do artigo científico

68,3

Relevância do periódico

Texto estar em português

Formato do texto completo ser em PDF

Texto ser pouco extenso

Texto estar em inglês

Outros fatores

TOTAL

100,0

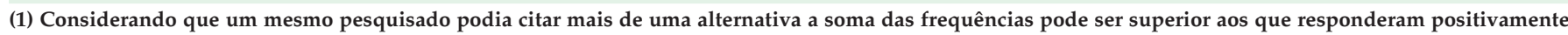
na primeira questão da tabela. 


\section{TABELA 4}

Avaliação da opinião sobre as assertivas expostas segundo grupos iniciante, intermediário e avançado

\section{Grupo de períodos}

\section{TOTAL}

\begin{tabular}{|c|c|c|c|c|c|c|c|c|}
\hline \multicolumn{2}{|c|}{$1^{\circ}$ ao $3^{\circ}$} & \multicolumn{2}{|c|}{$5^{\circ}$ ao $7^{\circ}$} & \multicolumn{2}{|c|}{$9^{\circ}$ ao $11^{\circ}$} & \multicolumn{2}{|c|}{ Grupo total } & \multirow[t]{2}{*}{ Valor de $\mathrm{p}$} \\
\hline $\mathbf{n}$ & $\%$ & $\mathrm{n}$ & $\%$ & $\mathbf{n}$ & $\%$ & n & $\%$ & \\
\hline 98 & 100,0 & 88 & 100,0 & 79 & 100,0 & 265 & 100,0 & \\
\hline
\end{tabular}

Tenho apoio familiar para exercer meus afazeres na faculdade

Discorda

Sem opinião

$\begin{array}{cccccccc}1 & 1,0 & 1 & 1,1 & 1 & 1,3 & 3 & 1,1 \\ 1 & 1,0 & 1 & 1,1 & 1 & 1,3 & 3 & 1,1 \\ 96 & 98,0 & 86 & 97,7 & 77 & 97,5 & 259 & 97,7\end{array}$

$\mathrm{p}^{(1)}=1,000$

Concorda

Costumo estudar em minha residência por conseguir concentração adequada/suficiente.

Discorda

Sem opinião

$\begin{array}{llllllll}16 & 16,3 & 10 & 11,4 & 7 & 8,9 & 33 & 12,5\end{array}$

Concorda

$\begin{array}{llllllll}1 & 1,0 & 5 & 5,7 & 6 & 7,6 & 12 & 4,5\end{array}$

Meu tempo de estudo é reduzido devido à distância entre minha

$\begin{array}{llllllll}81 & 82,7 & 73 & 83,0 & 66 & 83,5 & 220 & 83,0\end{array}$

moradia e a faculdade

Discorda

Sem opinião

$\begin{array}{cccccccc}38 & 38,8 & 47 & 53,4 & 48 & 60,8 & 133 & 50,2 \\ 5 & 5,1 & 3 & 3,4 & 5 & 6,3 & 13 & 4,9 \\ 55 & 56,1 & 38 & 43,2 & 26 & 32,9 & 119 & 44,9\end{array}$

Concorda

Tenho acesso a jornais/revistas científicas de meu curso de graduação

Discorda

$\begin{array}{llllllll}27 & 27,6 & 20 & 22,7 & 19 & 24,1 & 66 & 24,9 \\ 22 & 22,4 & 12 & 13,6 & 12 & 15,2 & 46 & 17,4 \\ 49 & 50,0 & 56 & 63,6 & 48 & 60,8 & 153 & 57,7\end{array}$

Concorda

Costumo pegar livros emprestados na biblioteca da faculdade para estudar

Discorda

Sem opinião

Concorda

$\begin{array}{cccccccc}5 & 5,1 & 22 & 25,0 & 34 & 43,0 & 61 & 23,0 \\ 3 & 3,1 & 4 & 4,5 & 8 & 10,1 & 15 & 5,7 \\ 90 & 91,8 & 62 & 70,5 & 37 & 46,8 & 189 & 71,3\end{array}$

Me disponho a custear conteúdos acadêmicos on-line pagos

Discorda

Sem opinião

Concorda

$\begin{array}{ccccccccc}60 & 61,2 & 75 & 85,2 & 52 & 65,8 & 187 & 70,6 & \mathrm{p}^{(2)}=0,003^{*} \\ 19 & 19,4 & 9 & 10,2 & 11 & 13,9 & 39 & 14,7 & \\ 19 & 19,4 & 4 & 4,5 & 16 & 20,3 & 39 & 14,7 & \end{array}$

\section{Só consigo estudar com disponibilidade de internet}

Discorda

Sem opinião

Concorda

$\begin{array}{cccccccc}28 & 28,6 & 42 & 47,7 & 31 & 39,2 & 101 & 38,1 \\ 14 & 14,3 & 6 & 6,8 & 5 & 6,3 & 25 & 9,4 \\ 56 & 57,1 & 40 & 45,5 & 43 & 54,4 & 139 & 52,5\end{array}$

\begin{tabular}{|c|c|c|c|c|c|c|c|c|}
\hline Percebo que estou menos atualizado se não estudar conteúdos on-line & & & & & & & 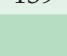 & $\mathrm{p}^{(2)}=0,017^{*}$ \\
\hline Discorda & 33 & 33,7 & 38 & 43,2 & 17 & 21,5 & 88 & 33,2 \\
\hline Sem opinião & 15 & 15,3 & 14 & 15,9 & 9 & 11,4 & 38 & 14,3 \\
\hline Concorda & 50 & 51,0 & 36 & 40,9 & 53 & 67,1 & 139 & 52,5 \\
\hline
\end{tabular}

Compartilho frequentemente informações e conteúdos on-line com meus colegas

Discorda

Sem opinião

$\begin{array}{llllllll}9 & 9,2 & 15 & 17,0 & 1 & 1,3 & 25 & 9,4\end{array}$

Concorda

$\begin{array}{llllllll}5 & 5,1 & 9 & 10,2 & 10 & 12,7 & 24 & 9,1\end{array}$

Tenho conhecimento da importância da prática baseada em evidências

Discorda

Sem opinião

$\begin{array}{llllllll}84 & 85,7 & 64 & 72,7 & 68 & 86,1 & 216 & 81,5\end{array}$

Concorda

$\begin{array}{cccccccc}2 & 2,0 & 1 & 1,1 & - & - & 3 & 1,1 \\ 5 & 5,1 & 5 & 5,7 & 2 & 2,5 & 12 & 4,5 \\ 91 & 92,9 & 82 & 93,2 & 77 & 97,5 & 250 & 94,3\end{array}$

(*) Associação significativa ao nível de 5,0\%

(1) Através do teste Exato de Fisher

(2) Através do teste Qui-quadrado de Pearson. 
linguística é um impedimento para uma consulta à literatura. Entretanto, cerca de $83 \%$ dos participantes que afirmaram utilizar bases de dados eletrônicas em sua rotina acadêmica de estudos não priorizam os textos escritos na língua inglesa em sua pesquisa. Portanto, infere-se que, na prática, ainda há certa resistência ao uso efetivo de artigos em inglês, podendo haver prejuízo à qualidade bibliográfica buscada pelo estudante de língua portuguesa, visto que, segundo Mouillete ${ }^{13}$, para usar integralmente as interfaces e recursos de busca é necessário possuir conhecimento e domínio de outros idiomas.

Sobre as atitudes dos acadêmicos quanto às plataformas de pesquisas preferenciais, observamos que um estudo americano ${ }^{14}$ realizado com 68 estudantes do terceiro ano do curso de Medicina mostrou que a plataforma de pesquisa UpToDate foi a segunda mais citada, com 51 (75\%) escolhas devido à confiança das informações e à rapidez da busca, perdendo apenas para o prontuário eletrônico de saúde. Diferentemente, sobre o UpToDate, plataforma de saúde baseada em evidências, na amostra deste estudo ( $p<0,001)$, apenas $31(11,7 \%)$ de todos os estudantes e $26(32,9 \%)$ dos que estão no terceiro ano do curso referiram identificação com esse meio de estudo eletrônico, demonstrando concordância com a preferência dos já comentados livros, online ou impressos. Embora esta preferência tenha sido proeminente perante outros meios de busca por informações, a amostra desta pesquisa referiu que 131 (49,4\%; $\mathrm{p}=0,002$ ) estudantes afirmaram não dispor de uma biblioteca de fácil acesso ou com acervo e serviços adequados disponíveis para a vida acadêmica.

Sobre a importância da prática em medicina baseada em evidências, um estudo qualitativo iraniano ${ }^{15}$ demonstrou, segundo a opinião de 40 estudantes de Medicina participantes, que se deve ter habilidades nesse tipo de fonte de pesquisa, estimando por informações atualizadas, com suporte educacional não somente de equipamentos eletrônicos, como também por atitudes positivas de interesse dos próprios estudantes a fim de aumentar o senso de autoconfiança e empoderamento. Já num estudo inglês ${ }^{16}, 15$ alunos de Medicina afirmaram que os recursos utilizados na busca por informação devem ser fáceis de usar, rápidos, ter qualidade e servir para diferentes propósitos, como numa rápida prescrição de medicamentos em estudos mais avançados de conhecimento, embora não houvesse consenso sobre uma fonte única que abrangesse todas essas qualidades. Nesse aspecto, 250 (94,3\%; p = 0,676) dos estudantes da amostra deste estudo concordaram sobre a relevância da prática baseada em evidências em sua rotina acadêmica, inclusive com 139 (52,5\%; p = 0,017) estudantes afirmando que se percebem menos atualizado ao deixarem de buscar por informações no âmbito online.
Em relação às formas de estudo com que os alunos mais se identificam, as assertivas mais elegidas na presente pesquisa foram "livros online (PDF, Word, Epub, etc.)", com 81,9\% de preferência, seguida por "livros em papel", com 68,3\%. Em busca de esclarecer hábitos de leitura de livros eletrônicos, uma pesquisa realizada pela Editora da Universidade de São Paulo estudou as vantagens que levaram os universitários a comprar ou acessar a versão online de um livro, em vez da versão impressa, e as opções mais relatadas foram "o livro eletrônico estava disponível gratuitamente em um site" (65,9\%) e "o livro impresso não existia na biblioteca" (30,1\%). Por outro lado, entre as principais desvantagens do uso do livro digital em comparação ao livro em papel, foram apontadas: "a leitura em tela é cansativa" (59,2\%) e "não poder fazer anotações ou grifar trechos a caneta ou a lápis na página" $(43,7 \%)^{17}$.

Comparando-se os grupos iniciante, intermediário e avançado com relação aos meios de estudo com que os estudantes mais se identificam, notou-se uma diferença daqueles que assinalaram apostilas de cursinho para residência $(28,7 \%)$, pois há um aumento significativo deste meio entre os grupos inicial $(8,2 \%)$ e intermediário $(26,1 \%)$ para o grupo avançado (57\%). Isto mostra que, com a aproximação do término do curso e a preparação para a realização das provas de residência, os estudantes tendem a utilizar apostilas de cursos preparatórios também como meio de estudo acadêmico.

Outro dado importante refere-se à discrepância de tempo dedicado, por semana, ao meio de estudo preferencial escolhido pelos estudantes. No grupo inicial, $48 \%$ se dedicam mais de 15 horas semanais, enquanto no grupo avançado a maioria dos estudantes $(50,6 \%)$ se dedica de cinco a dez horas semanais. Esse dado demonstra que o fator tempo interfere negativamente na dedicação dos estudantes à busca das informações nos períodos acadêmicos finais, o que talvez se justifique pela alta carga horária destinada às atividades práticas de estágio.

Também foi possível notar diferenças na questão relacionada ao uso dos livros em papel, pois, embora se trate do segundo meio de estudo de maior preferência do grupo geral de alunos, houve discrepância entre os grupos inicial, intermediário e avançado: nos grupos inicial e intermediário, o maior percentual de alunos $(82,7 \%$ e $71,6 \%$, respectivamente) se identificava com esse meio; já no grupo avançado, esse valor decresce substancialmente: apenas $46,8 \%$ dos estudantes afirmaram se identificar com o mesmo. $\mathrm{O}$ decréscimo entre os grupos ocorre segundo o avançar dos períodos, tendo o grupo intermediário menor percentual que o grupo inicial. Observa-se também que essa relação é inversa quando se trata da assertiva relacionada à identificação com as plataformas de 
saúde baseadas em evidências (Dynamed/UpToDate): à medida que os grupos avançam de período, a porcentagem de alunos que se identifica com esse meio aumenta: no grupo inicial, a percentagem foi de apenas $1 \%$ dos alunos; já no grupo intermediário, esse valor é de 4,5\% dos estudantes, chegando a aumentar de forma considerável (aproximadamente sete vezes maior) quando se trata do grupo avançado, em que 32,9\% dos alunos se identificam com esse meio. Tais dados sugerem que muitos dos usuários dos livros em papel, com o avançar do curso, passam a utilizar as plataformas online baseadas em evidências em busca de atualizações mais recentes e da confiabilidade das informações procuradas.

Nota-se, portanto, que ao longo do curso variam as questões relativas aos meios para buscar informação e à maturidade e experiência de como e onde estudar. Tais informações são imprescindíveis para analisar a evolução dos estudantes ao longo do curso e possíveis hábitos que venham a adquirir na vida profissional.

\section{CONCLUSÃO}

A maior parte dos estudantes tem acesso à informação científica, sendo os livros online os meios de estudo prioritários. A utilização de resumos acadêmicos dos colegas, sites e livros impressos diminui com o avançar do curso, enquanto as plataformas de saúde baseadas em evidências têm destaque entre os matriculados a partir do nono período, o que sugere incorporação científica ao longo da formação e necessidade de usar informação mais atualizada e maior rigor metodológico. Porém, mesmo entre os que afirmam se valer de artigos científicos em seus estudos, as estratégias de busca foram aprendidas com a prática e, portanto, não há uso adequado de descritores ou formas de busca com filtros mais específicos.

É preciso oferecer formação em recursos digitais de forma constante ao corpo discente e docente de modo a colaborar com a formação de uma cultura acadêmica centrada no conhecimento científico atualizado, o que trará consequências para a incorporação desta prática no cotidiano profissional, qualificando a prática médica futura.

Diante da relevância da temática abordada, sugere-se avaliar em estudos futuros se o comportamento e a opinião de estudantes inseridos numa metodologia tradicional de ensino são semelhantes ou diferem dos resultados encontrados na presente pesquisa.

\section{REFERÊNCIAS}

1. Wilson TD. Human Information Behavior. Inf. Sci. [online]. 2000. 3(2) [capturado 30 jul. 2018]. Disponível em: http:/ / inform.nu/Articles/Vol3/v3n2p49-56.pdf.
2. Felder RM, Brent R. Understanding student differences. J. Eng. Educ. [online]. 2015. 94(1) [capturado 30 jul. 2018]; 57-72. Disponível em: http://www4.ncsu.edu/unity/ lockers/users/f/felder/public/Papers/Understanding_ Differences.pdf

3. Miranda SMD, Pires MMDS, Nassar SM, Silva CAJD. Construção de uma escala para avaliar atitudes de estudantes de medicina. Rev. Bras. Educ. Med. [online]. 2009. 33(1) [capturado 30 jul. 2018]; 104-110. Disponível em: https:/ /doi.org/10.1590/S0100-55022009000500011.

4. Navarro-Hernández N, Zamora-Silva J. Factores que facilitan u obstaculizan El aprendizaje basado en problemas en grupo pequeño, vistos por los estudiantes de la Facultad de Medicina de la Universidad de La Frontera, Temuco, Chile. Iatreia. [online]. 2016. 29(2) [capturado 06 jul. 2018]; 113-122. Disponível em: http://pesquisa.bvsalud.org/ portal/resource/pt/lil-785519.

5. Silveira MSM. A informação científica na prática médica: estudo do comportamento informacional do médico-residente. Salvador; 2005. Mestrado [Dissertação] Universidade Federal da Bahia. Disponível em: http:/ /www. gmbahia.ufba.br/i HYPERLINK "http://www.gmbahia. ufba.br/index.php/gmbahia/article/viewFile/367/356" HYPERLINK "http://www.gmbahia.ufba.br/index. php/gmbahia/article/viewFile/367/356" HYPERLINK "http: / / www.gmbahia.ufba.br/index.php/gmbahia / article/viewFile/367/356" HYPERLINK "http://www. gmbahia.ufba.br/index.php/gmbahia/article/viewFile/367/356" HYPERLINK "http://www.gmbahia.ufba. br/index.php/gmbahia / article / viewFile/367/356" HYPERLINK "http://www.gmbahia.ufba.br/index. php/gmbahia/article/viewFile/367/356" HYPERLINK "http:/ / www.gmbahia.ufba.br/index.php/gmbahia / article/viewFile/367/356"ndex.php/gmbahia/article/ viewFile/367/356.

6. Chumley HD, Dobbie AE, Jr JED. Case-based exercises fail to improve medical students' information management skills: a controlledtrial. BMC Med. Educ. [online]. 2006. 6. [capturado 20 jul. 2018]; 14. Disponível em: https:/ / www. ncbi.nlm.nih.gov/pmc/articles/PMC1420296/.

7. Mesko B, Gyorffy Z, Kollar J. Digital Literacy in the Medical Curriculum: A CourseWith Social Media Tools andGamification. JMIR Med. Educ. [online]. 2005. 1(2) [capturado 30 jul. 2018]. Disponível em: https://doi.org/10.2196/ mededu.4411.

8. Peterson MW, Rowat J, Kreiter C, Mandel J. Medical students' use of information resources: is the digital age dawning? Acad. Med. [online]. 2004. 79(1) [capturado 30 jul. 
2018]; 89-95. Disponível em: https://www.ncbi.nlm.nih. gov/pubmed/14691004.

9. Gaitsgory O, Burgess A, Mellis C. Opinionpiece: 'Medical students - learning from text book sorelectronic media?' J. Paediatr. Child Health. [online]. 2013. 49(9) [capturado 30 jul. 2018]. Disponível em: ht HYPERLINK "http:/ / onlinelibrary.wiley.com/doi/10.1111/jpc.12180/ full" HYPERLINK "http://onlinelibrary.wiley.com/ doi/10.1111/jpc.12180/full" HYPERLINK “http://onlinelibrary.wiley.com/doi/10.1111/jpc.12180/full" HYPERLINK "http:/ / onlinelibrary.wiley.com/doi/10.1111/ jpc.12180/full" HYPERLINK “http://onlinelibrary. wiley.com/doi/10.1111/jpc.12180/full" HYPERLINK "http:/ / onlinelibrary.wiley.com/doi/10.1111/jpc.12180/ full" HYPERLINK "http://onlinelibrary.wiley.com/ doi/10.1111/jpc.12180/full”tp: / / onlinelibrary.wiley. com/doi/10.1111/jpc.12180/full

10. Parve S, Ershadi A, Karimov A, Dougherty A, Ndhlovu $\mathrm{CE}$, Chidzonga MM, et al. Access, attitudes and training in information Technologies and evidence-based medicine among medical students at University of Zimbabwe College of Health Sciences. Afr Health Sci [online]. 2016. 16(3) [capturado 20 jul. 2018]; 860-865. Disponível em: https:/ / www.ncbi.nlm.nih.gov/pubmed/27917222.

11. Pereira IS, Diniz Júnior J. Competência em informação no ensino superior: um estudo com discentes do curso de graduação em Medicina da Escola Multicampi de Ciências Médicas do Rio Grande do Norte. Ver Eletrônica de Comunicação, Informação e Inovação em Saúde [online]. 2017. 11(1) [capturado 20 jul. 2018]; Disponível em: https:// www.reciis.icict.fiocruz.br/index.php/reciis/article/ view/1202.

12. Pereira Jr A. A publicação cientifica na atualidade. J Vasc Bras. [online]. 2007. 6(4) [capturado 18 ago. 2018]; 307-308. Disponível em: http://www.scielo.br/pdf/jvb/v6n4/ v6n4a02.pdf

13. Mouillet E. Languagebarriersandbibliographicretrievaleffectiveness: use of MEDLINE byFrenc-speakingendusers. Bull MedLibr Assoc. [online]. 1999. 87(4) [capturado 20 jul. 2018]; 451-5. Disponível em: https://www.ncbi.nlm. nih.gov/pub HYPERLINK "https://www.ncbi.nlm.nih. gov/pubmed/10550030" HYPERLINK "https://www. ncbi.nlm.nih.gov/pubmed/10550030" HYPERLINK "https:/ / www.ncbi.nlm.nih.gov/pubmed/10550030" HYPERLINK "https://www.ncbi.nlm.nih.gov/pubmed/10550030" HYPERLINK "https://www.ncbi. nlm.nih.gov/pubmed/10550030" HYPERLINK "https: / / www.ncbi.nlm.nih.gov / pubmed / 10550030" HYPERLINK "https://www.ncbi.nlm.nih.gov/ pubmed/10550030"med/10550030.

14. Twiss-Brooks AB, Andrade Jr R, Bass MB, Kern B, Peterson J, Werner DA. A day in thelifeofthird-year medical students: using na ethnographicmethodtounderstandinformationseekingand use. J MedLibr Assoc. [online]. 2017. 105(1) [capturado 20 jul 2018]; 12-19. Disponível em: https://www.ncbi.nlm.nih.gov/pmc/articles / PMC5234461/.

15. Ghojazadeh M, Hajebrahimi S, Azami-Aghdash S, Pournaghi Azar F, Keshavarz, M, Naghavi-BehzadM,Hazrati H. Medical students' attitudesonandexperienceswithevidence-basedmedicine: a qualitativestudy. J EvalClinPract. [online]. 2014. 20(6) [capturado 19 jul 2018]; 779-85. Disponível em: https://onlinelibrary.wiley.com/doi/ pdf/10.1111/jep.12191.

16. Brennan N, Edwards S, Kelly N, Miller A, Harrower L, Mattick K. Qualifieddoctorand medical students' use ofresources for accessinginformation: whatisusedandwhy? Health Info Libr J. [online]. 2014. 31(3) [capturado 19 jul 2018]; 204-14. Disponível em: https://www.ncbi.nlm.nih. gov/pubmed/25041386.

17. Martins Filho P. Livros Eletrônicos na Universidade. Edusp - Editora da Universidade de São Paulo. [online]. 2015. [capturado 18 ago. 2018]. Disponível em: http:// www.edusp.com.br/pesquisa/Livros_eletronicos_na_ universidade.pdf

\section{CONTRIBUIÇÃO DOS AUTORES}

Concepção e desenho do estudo: Suélem Barros de Lorena. Aquisição, análise ou interpretação dos dados: Mateus de Melo Andrade, Lycia Siqueira Vilela

Redação do manuscrito: Ângela Melo de Holanda Cavalcanti, Suélem Barros de Lorena.

Revisão crítica do conteúdo intelectual: José Eudes de Lorena Sobrinho, Suélem Barros de Lorena.

\section{CONFLITO DE INTERESSES}

Não houve conflito de interesses dos autores no desenvolvimento deste trabalho.

\section{ENDEREÇO PARA CORRESPONDÊNCIA}

Avenida Mal. Mascarenhas de Morais, 4861, Imbiribeira, Recife-PE, CEP: 51.150-000. 